\title{
Controlling the differential resistance of axial blown arcs
}

\section{Journal Article}

Author(s):

Bort, Lorenz (D); Vonesch, Miriam; Franck, Christian (D)

Publication date:

2019-11-06

Permanent link:

https://doi.org/10.3929/ethz-b-000361410

Rights / license:

In Copyright - Non-Commercial Use Permitted

Originally published in:

Journal of Physics D: Applied Physics 52(45), https://doi.org/10.1088/1361-6463/ab34e0 
ACCEPTED MANUSCRIPT

\section{Controlling the differential resistance of axial blown arcs}

To cite this article before publication: Lorenz Stefan Johannes Bort et al 2019 J. Phys. D: Appl. Phys. in press https://doi.org/10.1088/1361$\underline{6463 / \mathrm{ab} 34 \mathrm{e} 0}$

\section{Manuscript version: Accepted Manuscript}

Accepted Manuscript is "the version of the article accepted for publication including all changes made as a result of the peer review process, and which may also include the addition to the article by IOP Publishing of a header, an article ID, a cover sheet and/or an 'Accepted Manuscript' watermark, but excluding any other editing, typesetting or other changes made by IOP Publishing and/or its licensors"

This Accepted Manuscript is ( 2019 IOP Publishing Ltd.

During the embargo period (the 12 month period from the publication of the Version of Record of this article), the Accepted Manuscript is fully protected by copyright and cannot be reused or reposted elsewhere.

As the Version of Record of this article is going to be / has been published on a subscription basis, this Accepted Manuscript is available for reuse under a CC BY-NC-ND 3.0 licence after the 12 month embargo period.

After the embargo period, everyone is permitted to use copy and redistribute this article for non-commercial purposes only, provided that they adhere to all the terms of the licence https://creativecommons.org/licences/by-nc-nd/3.0

Although reasonable endeavours have been taken to obtain all necessary permissions from third parties to include their copyrighted content within this article, their full citation and copyright line may not be present in this Accepted Manuscript version. Before using any content from this article, please refer to the Version of Record on IOPscience once published for full citation and copyright details, as permissions will likely be required. All third party content is fully copyright protected, unless specifically stated otherwise in the figure caption in the Version of Record.

View the article online for updates and enhancements. 


\title{
Controlling the Differential Resistance of Axial Blown Arcs
}

\author{
L.S.J. Bort ${ }^{1}$, M. Vonesch ${ }^{1}$, C.M. Franck ${ }^{1}$ \\ ${ }^{1}$ Power Systems and High Voltage Laboratories, ETH Zurich, Physikstr. 3, 8092 Zurich, Switzerland \\ E-mail: lorenz.bort@alumni.ethz.ch
}

\begin{abstract}
Transfer switches for future HVDC systems can be based on passive oscillation principle to create a current zero crossing. The principle relies on a negative differential arc resistance $\mathrm{d} u / \mathrm{d} i$ of the mechanical switch.Present investigation aims to understand and quantify under which conditions the $\mathrm{d} u / \mathrm{d} i$ is negative. For this, results from electrical arc measurements are compared to results from a $1 \mathrm{D}$ arc model. Based on this, the authors conclude that ablation is not the reason for non-negative $\mathrm{d} u / \mathrm{d} i$. In fact it is rather the location of the smallest arc radius which determines the negative characteristic. As soon as the turbulent shear layer is thick enough compared to the total arc diameter that it influences the temperature at the arc center, the $\mathrm{d} u / \mathrm{d} i$ turns negative. The current at which this happens scales with $p^{2 / 3}$, can be influenced by the nozzle shape and is rather insensitive to the chosen type of blowing gas.
\end{abstract}

\section{Introduction}

Future HVDC systems, especially meshed grids, require more DC switchgear than today's installations. Circuit breakers that can interrupt fast rising fault currents have received much attention recently, but there are many cases where speed is less critical, and simpler devices can be used. The joint working group A3/B4.34 of CIGRE coined the term "transfer switch" in their technical brochure 683 [1]. These switches are used in all cases, where a load current needs to be interrupted or commutated, against a driving voltage between a few $\mathrm{kV}$ and the system voltage. Metal return transfer switches (MRTS) are one example, but also cases where one of several parallel lines or a non-faulty but loaded line are disconnected fall in this category.

For those cases, switches relying on passive oscillation are a good choice, since they do not posses the drawbacks of using power semiconductors. The only parts needed are a mechanical gas blast circuit breaker (CB), as well as a parallel LC branch and an arrester bank. This concept has already been described in the last century, for example by Lee et.al. [2]. As soon as the CB opens, an oscillating current in the loop consisting of $\mathrm{L}, \mathrm{C}$ and $\mathrm{CB}$ is started, which is superimposed on the nominal DC current flowing through the breaker. If the differential resistance of the $\operatorname{arc} \mathrm{d} u / \mathrm{d} i$ is negative, this oscillating current is amplified, until it reaches the same amplitude as the DC current. This creates a current zero in the CB, allowing it to clear and commutate the current into the LC-branch first, and subsequently into the arrester bank. The only active component is the mechanical drive, which makes this concept very attractive.

The performance of the passive oscillation concept depends on the arc voltage, specifically on the fact that with rising current the voltage drop decreases. This is the case in conventional gas blast AC breakers, at least up to a certain current. As long as the differential resistance $\mathrm{d} u / \mathrm{d} i$ plus the parasitic resistance of the LC branch is negative, damping of the oscillation is negative and the oscillation grows. If the $u(i)$ curve of the arc could be altered to be more steeply falling at higher/current amplitudes, the time to current zero, as well as the/maximum interruptable current can be improved. For existing applications, like MRTS, an optimized interrupter could also enable the use of smaller values of $\mathrm{L}$ and $\mathrm{C}$, leading to a reduction of both cost and footprint.

Most of the research on switching arcs in the past was focused on the conditions around current zero, to improve the breaking performance of AC breakers. In contrast, for the optimization of passive oscillation breakers, behavior in the intermediate current range is important, where the arc is stabilized by the axially gas flow, and not dominated by nozzle wall ablation. Quite some literature, for example by Hermann, Kogelschatz et.al.[3], covers this regime. But the understanding which external parameters determine the $u(i)$ curve and especially the derivative $\mathrm{d} u / \mathrm{d} i$ is still poor. In axially blown arcs, the main cooling mechanisms are convection and radiation, while heat conduction is negligible. How exactly those cooling terms scale with current, and which of them is responsible for the needed negative $\mathrm{d} u / \mathrm{d} i$ is still not well understood. This knowledge would enable optimization of the interrupter specifically for passive oscillation.

Previous work by the author includes investigating the influence of nozzle wall ablation [4] and determining which axial segment of the arc contributes how much to the total arc voltage [5]. Both papers indicated that ablation onset might be unfavorable for the $u(i)$ characteristics, and that the voltage drop of upstream segments is higher and additionally has more 
negative $\mathrm{d} u / \mathrm{d} i$.

Additional to the measurements of Hermann et. al. [3], there was theoretical work done by Tuma, Lowke and Ludwig [6, 7]. Axially blown arcs are quite dynamic and fluctuate in all three dimensions. Nevertheless, simple 1D models, which assume cylindric symmetry, are worth using, at least to gain some qualitative understanding of the physical processes.

The aim of the present contribution is to understand why arcs have a negative differential resistance $\mathrm{d} u / \mathrm{d} i$ at low currents, and flat or rising $u(i)$ behavior at higher currents. The focus lies on empirical experiments, but they are combined with a theoretical model.

\section{Setup}

The experimental setup used is almost identical to the setup described in [5]. A model circuit breaker with nozzle made from PMMA is used, in a single flow configuration with copper-tungsten electrodes. The exact geometry is shown in figure 1. Air is used as a blowing gas if not noted otherwise. The gas is fed to the nozzle inlet at a controlled pressure from storage canisters, i.e. independent of contact movement. Only small changes were implemented compared to previous work $[4,5,8]$. Notably, a lid was attached to the downstream pin, that allowed to close the nozzle before experiments, and therefore enable the use of other gases, without air entering the system before the upstream valves are opened.

In order to change the inlet conditions of the blowing gas to change the level of turbulence, some pertubators were added for one measurement series. Three "flow straightners", hive-like grids of different length and hole diameter, that should help dissipate turbulent patterns and homogenize the inlet flow. They are shown in figure 2. To introduce more turbulent inlet conditions, perforated metal plates were used, which block $61 \%$ and $68 \%$ of the cross section respectively, and introduce additional turbulence. It must be noted, that it cannot be expected to achieve laminar flow in the nozzle inlet. The density and velocity of the gas are too high, even with the smallest hole size of $3 \mathrm{~mm}$ the Reynolds number is around 50'000. However, inserting something in the inlet was an easy way to test if turbulence can be influenced, and if that has an impact on the arc behaviour.

\section{Method}

\subsection{Experimental work}

Determination of the stationary arc voltage is done as described in more detail in previous work $[5,8]$,

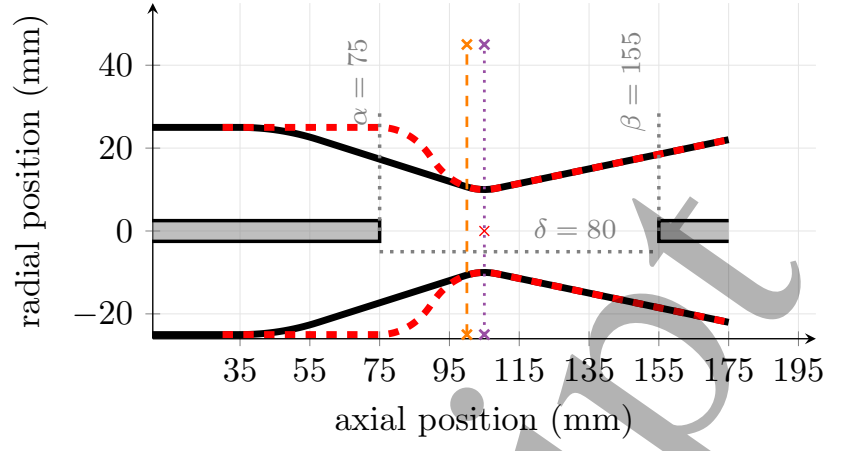

Figure 1: Nozzle setup, gas flow is from left to right. $\alpha$ indicates upstream contact position, $\beta$ the downstream contact position. The throat position is at $105 \mathrm{~mm}$ (red cross). The black solid line indicates the nozzle contour which was used for the majority of experiments. Converging and diverging parts are both conical, with half angle of $15^{\circ}$ and $10^{\circ}$ respectively. The red dashed line indicates the contour of a nozzle variant with a shorter converging segment with up to $45^{\circ}$ half angle, leading to higher acceleration in that segment. The purple line indicates the throat position, and the orange line is the position where the arc radius is evaluated, as shown in figure 4 .

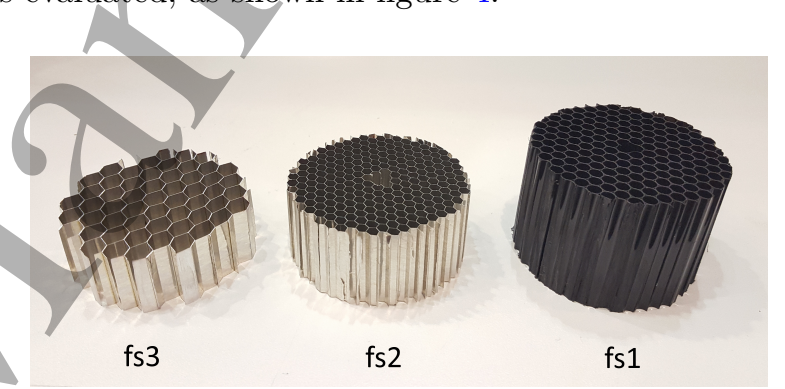

Figure 2: Flow straighteners 1 to 3 , used in the inlet condition variation, as described in section 5 and figure 11. Diameter of the holes are (from left to right) $7 \mathrm{~mm}, 3.1 \mathrm{~mm}$ and $4 \mathrm{~mm}$. The length in flow direction is $19 \mathrm{~mm}, 25 \mathrm{~mm}$ and $38 \mathrm{~mm}$ respectively.

using the Flexible Pulsed Current Source (FPDCS), described in [9]. A steady state arc is established by opening the interrupter contacts and blowing system valves, which takes roughly $70 \mathrm{~ms}$ and is done during a period of low current (less than 200 A). For the determination of the static $u(i)$ arc characteristic, a triangular current pulse is used, with linear rise to $1650 \mathrm{~A}$ in about $5 \mathrm{~ms}$ followed by a linear decrease to $0 \mathrm{~A}$ which also takes around $5 \mathrm{~ms}$. All the illustrated measurements are taken in the same configuration, with the upstream and downstream contacts stationary as indicated in figure 1 . The gas flow is quasi static with the pressure at the nozzle inlet being a reasonably constant value as stated in each graph. As discussed 
in [8], all voltages in the figures refer to the median voltage, extracted from the waveforms of two or three identical experiments. Where errorbars are plotted, they show the 5- and 95-percentile.

\subsection{D Model}

To get a rough estimation on the cooling mechanisms of the arc column, a simplified $1 \mathrm{D}$ model is used. It was inspired strongly by the work of Lowke, Ludwig and Tuma [6,7], which assumes cylindrical symmetry and two radial zones only. In the center there is hot arc plasma, with uniform properties in radial direction. Around that, there is cold gas, also with uniform properties in radial direction. The cold gas flow is not influenced by the arc, so there is no clogging of the throat and no heating of the cold gas. With these assumptions, analytical formulas for compressible flow can be used to determine all fluid- and thermodynamic variables of the cold gas zone as function of axial position. The key assumption of Lowke's model is the absence of a radial pressure gradient. If this holds, the cold flow determines the mach number of the hot plasma zone as well. With mach number and pressure fixed by the cold flow, it is possible to solve for arc temperature and radius, by dividing the problem into short axial segments $(0.1 \mathrm{~mm}$ long) and solving the energy balance at each axial position along the arc axis. The required steps are explained briefly in the following.

First, the model uses the isentropic flow relations to determine the properties of the cold flow along the nozzle. Input parameters are: nozzle shape, inlet pressure and exit pressure, also needed is the heat capacity ratio $\gamma$, which is 1.4 for air.

Second, the plasma is modelled. This requires more detailed material data, which is given by D'Angola [10, 11]. This data set is valid up to $60000 \mathrm{~K}$ and 100 bar, and includes all values needed, namely specific enthalpy, electrical conductivity and density of air as function of pressure and temperature. For each arc segment, there are assumed to be three terms: ohmic heating (equation 1), radiation cooling (equation 2) and convective cooling (equation 3) including the axial energy flow by mass transport (equation 4).

$P_{\text {ohmic }}=i^{2} \cdot \frac{l}{\sigma A}$

$P_{\text {rad }}=\epsilon_{\text {net }} \cdot l A$

$P_{\text {conv }}=\dot{H}_{\text {out }}-\dot{H}_{\text {in }}$

$\dot{H}_{\text {out }}=h(p, T) \cdot \dot{m}(p, T, A)=h M c \rho A$

Throughout this manuscript, $A$ denotes the cross sectional area of an axial arc segment of length $l$, where $l$ is short enough for all variables to be homogeneous in axial direction. For the following results, $l=0.1 \mathrm{~mm}$ was used. The specific enthalpy $h$, the density $\rho$, the electric conductivity $\sigma$ and the speed of sound $c$ are all material parameters which depend only on local temperature $T$ and pressure $p$. The plasma mass flow out of the segment is $\dot{m}$, and $M$ denotes the local mach number of the flow, whereas $c$ is the local speed of sound. The convective cooling power of one segment is therefore equal to the enthalpy flow out (downstream) minus the enthalpy flow into the segment (from upstream). This can be either due to a temperature increase in that segment, or an increased mass flow due to radial inflow of cold gas. Both of these effects are combined in equations 3 and 4 .

The radiation losses are modelled to be proportional to the plasma volume, and dependence on temperature was implemented using net emission coefficients $\epsilon_{\text {net }}$ as published by Aubrecht and Bartlova [12]. Specifically, the data for $1 \mathrm{~mm}$ arc diameter was used, and pressure dependency was accounted for by rescaling $\epsilon_{n e t}$ linearly with local density.

As already mentioned, $p$ and $M$ are given by the analytical cold flow calculations, the current $i$ is fixed as well as the enthalpy flow $\dot{H}_{i n}$ into the segment from the segment upstream. For a given segment, the only free variables are plasma temperature $T$ and cross sectional area $A$ (i.e. arc radius).

If one assumes there are no other cooling terms except convection and radiation, those two have to be equal to the ohmic heating.

$P_{\text {ohmic }}=P_{\text {rad }}+P_{\text {conv }}$

This energy balance equation reduces the free parameters to one. For each plasma temperature, there is only one possible arc area that fulfills equations (1) to (5), and vice versa. To get a unique solution for $T$ and $A$, the combination is chosen which minimises the voltage drop over that segment. This follows the principle of least action and is similar to the arguments in [13]. Temperature is one of the free variables, and therefore density $\rho$ as well as specific enthalpy $h$ in equation (4) have to be always recalculated for each change of $T$ while searching the solution.

Since the enthalpy outflow of one segment is the inflow of the next, the global solution can be found by iterating the individual segments from upstream to downstream, while $\dot{H}_{\text {in }}$ of one segment is equal to $\dot{H}_{\text {out }}$ of the segment directly upstream. Figures 3, 4, 5, and 6 show results for one set of initial conditions, namely a current of $1000 \mathrm{~A}$, and inlet pressure as indicated.

Figure 3 shows the ohmic heating power, and how it is split between convection and radiation cooling. In the upstream part of the nozzle, the radiation term dominates. Only in the segment right around the throat the convection term gets slightly larger than the radiation term. This disagrees with the results

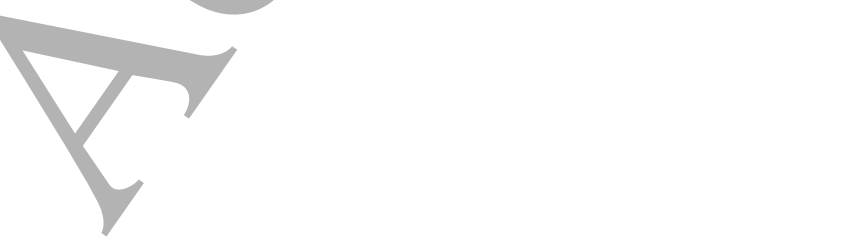


from [14], but agrees with the results from [15] who show a radially resolved energy balance at an axial position close to the throat. Downstream of the throat position (at $105 \mathrm{~mm}$, indicated by red dashed line), the convection term in this implementation becomes negative. The temperature goes to the upper bound of $60000 \mathrm{~K}$, and the plasma radius decreases. This effectively means that the solution found by the algorithm reduces the plasma mass flow for the downstream positions, and heats the remaining plasma by ejecting cold gas (less enthalpy flows out of segment than in). This clearly violates the second law of thermodynamics, and therefore the downstream segment was not used in the evaluation. This flaw of the model was tolerated, since previous work showed that the converging segments are the ones which contribute most to the overall arc voltage [5], and the downstream segments are not of high interest.

Figures 4 and 5 show the plasma radius and temperature respectively, as calculated by the model. There is little variation in the converging segment, only around the throat there are significant changes.

Figure 6 shows the electric field as function of axial position, which is in line with the results of [5]. The highest field is around the throat, with a gradual increase towards that position in the upstream part. In agreement with [6,7], this model results in a currentindependent arc voltage characteristic. The authors of those papers discuss that the negative differential arc resistance, which is experimentally observed for low currents, results from the effect of turbulence that becomes important when the arc radius reaches the size of the turbulent shear layer at the arc boundary. The effect of turbulence is not modelled here, but by comparing the modelled arc radius with the measured differential arc resistance, we can still estimate the current level at which this effect should become important.

Since different measurement series were performed, the authors decided to group the results and discussion according to the effects that were investigated. Section 3 describes the ablation measurements, section 4 discusses the influence of inlet pressure, section 5 is about inlet conditions and lastly section 6 shows measurements with different gases. Each section has its own Results and Discussion subsection.

\section{Ablation}

\subsection{Results}

Prior to this work, the authors investigated the influence of nozzle wall ablation on the arc voltage [4]. The results showed correlation between onset of measureable ablation and $\mathrm{d} u / \mathrm{d} i$ becoming zero or positive. To confirm causality, a material with as

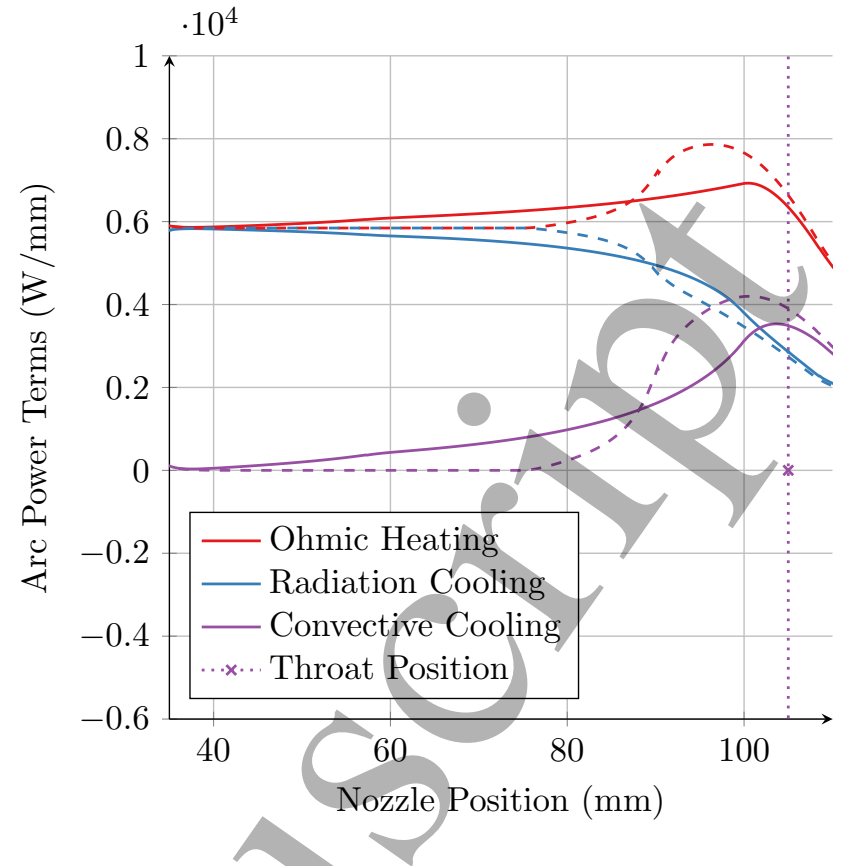

Figure 3: Heating and cooling terms according to 1D model simulation, for $1000 \mathrm{~A}$ and 7 bar inlet pressure. Solid lines show the results of the conical nozzle contour (black solid line in figure 1), dashed lines were obtained for the shorter acceleration profile (red dashed line in figure 1). It is/clear that for most of the converging part the radiation losses dominate, only a few $\mathrm{mm}$ in front of the throat $(105 \mathrm{~mm})$ - where acceleration is highest - the convection term briefly contributes a significant fraction to the cooling. In the diverging part, the convection term becomes negative and the model can no longer be applied.

little ablation as possible was searched. The company Brandenburger Isoliertechnik offers ablation resistant composite material, which was tested. Changing the nozzle material from PMMA to that material (trade name S5000) did not change the $u(i)$ curves significantly. Figure 7 shows virtually identical behavior, compared to PMMA for three different inlet pressures and air as blowing gas. Additional ablation measurements using weighing and scanning the nozzle contour as described in [4] showed that $55000 \mathrm{did}$ loose weight similarly to PMMA, although the nozzle contour did not change as much.

\subsection{Discussion}

The measurements with $S 5000$ and PMMA nozzles clearly show that ablation rates up to $1600 \mathrm{~A}$ are not high enough to influence the gas flow or the arc voltage significantly. This can also be justified by pressure measurements, which are not shown, that indicate that the upstream pressure does not change much with 


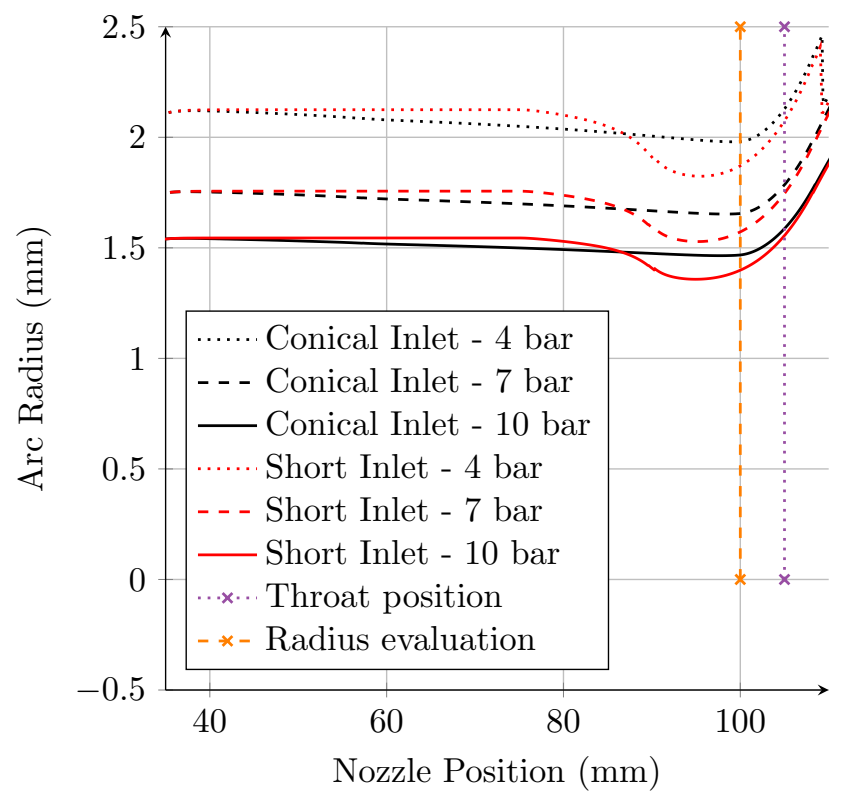

Figure 4: Plasma radius for $1000 \mathrm{~A}$ and three pressures, as determined by $1 \mathrm{D}$ model simulations. The throat position is indicated by the red dotted line, and the position used for the radius evaluation is indicated in orange.

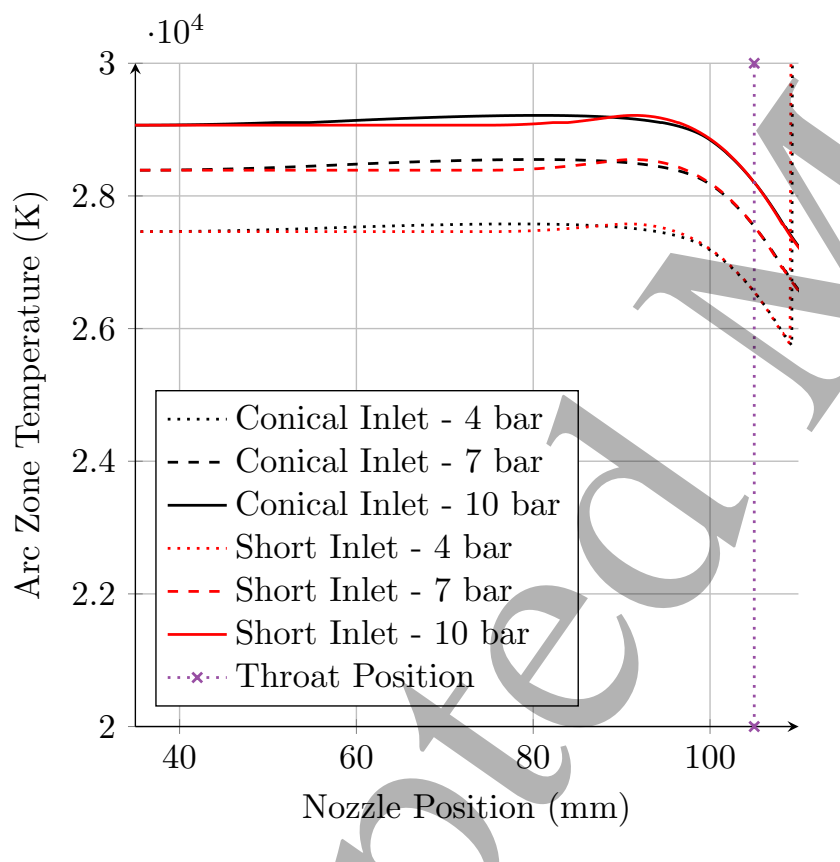

Figure 5: Arc zone temperature, as calculated by 1D model simulations. It can be seen how the temperature drops in the converging section, until the model fails shortly downstream of the throat and runs into the upper boundary at $60000 \mathrm{~K}$

changing current. If the mass flow of ablated wall material would be significant compared to the mass

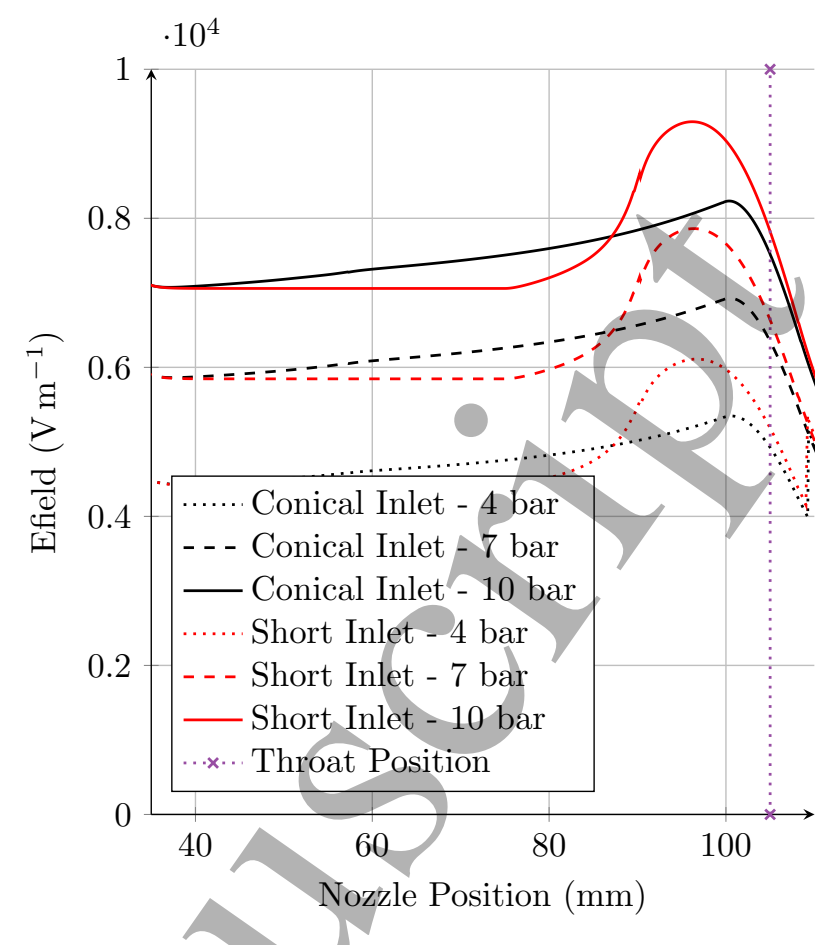

Figure 6: Electric field simulated by 1D model, for three inlet pressures and a current of $1000 \mathrm{~A}$. However, as explained in section 4.2 , the resulting voltage is independent of current, so these curves result for any input current.

flow of the blowing gas, this would lead to drastically rising pressures upstream as well as at the nozzle throat, where sensors are installed. This is not the case. Visual inspection of the $S 5000$ nozzles indicated charring of the material. Mass loss is most probably due to evaporation of volatile parts of the material, while simultaneously the charred material kept its shape. In general, it proved more difficult to determine ablation rates for $S 5000$ than for PMMA. The weight of $S 5000$ nozzles did change overnight, probably due to moisture ingress and drying processes, and the ablated surface was cracked and eroded by pieces breaking off, rather than homogenious ablation. This made the determination of ablation rates by measureing changes in geometry more challenging than it already is for PMMA.

Although the results are not as obvious as expected, the authors still believe that ablation is not the main driver for the differential resistance to become positive. Mainly because the onset current above which measureable ablation occurs is independent of pressure[4], whereas the transition point where $u(i)$ changes from falling to flat/rising is clearly pressure dependent, as shown in section 4 . 


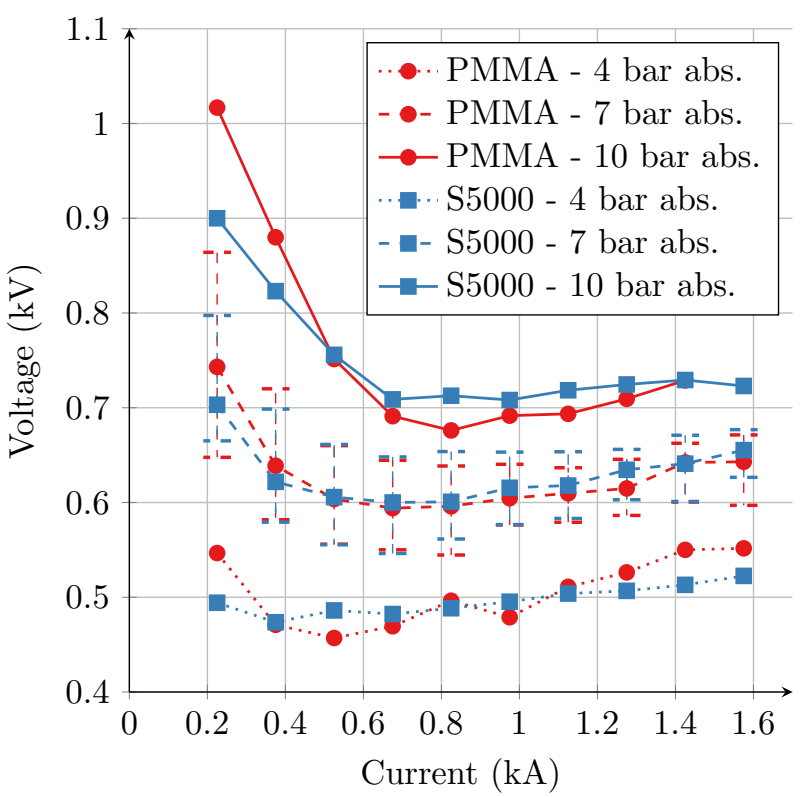

Figure 7: Measured arc voltage as function of current for experiments with different nozzle materials and different inlet pressures. Each point indicates the median voltage, calculated from at least $1 \mathrm{~ms}$ worth of data, as decribed in [8]. For the 7 bar abs. data sets, errorbars were included which show the 5- and 95-percentile of the arc voltage, giving an indication of the maximum fluctuation amplitude. Blowing gas was air.

\section{Inlet Pressure}

\subsection{Experimental Results}

Previous work showed that the upstream pressure of the blowing gas has a considerable influence on the $u(i)$ behavior [16]. This was confirmed by the experiments, as shown in figure 7. Higher inlet pressures move the $u(i)$ curve up, i.e. increase the overall arc voltage. Additionally, the transition point where $\mathrm{d} u / \mathrm{d} i$ becomes zero or even positive is shifted towards higher currents, as shown in figure 8. For air at $4 \mathrm{bar}, \mathrm{d} u / \mathrm{d} i$ is positive above about $450 \mathrm{~A}$, whereas at 10 bar, the voltage falls until $800 \mathrm{~A}$, and starts rising above $1000 \mathrm{~A}$. Additionally it shows differential resistance values between $-1 \Omega$ and $0.2 \Omega$, with higher pressure yielding more negative values for each current value.

\subsection{Discussion \& comparison with $1 D$ model}

For the passive oscillation to work, the sum of differential resistance of the arc plus the parasitic resistance of the LR-branch needs to be negative, to amplify the oscillating current. Figures 7 and 8 clearly show that increasing the inlet pressure is favourable

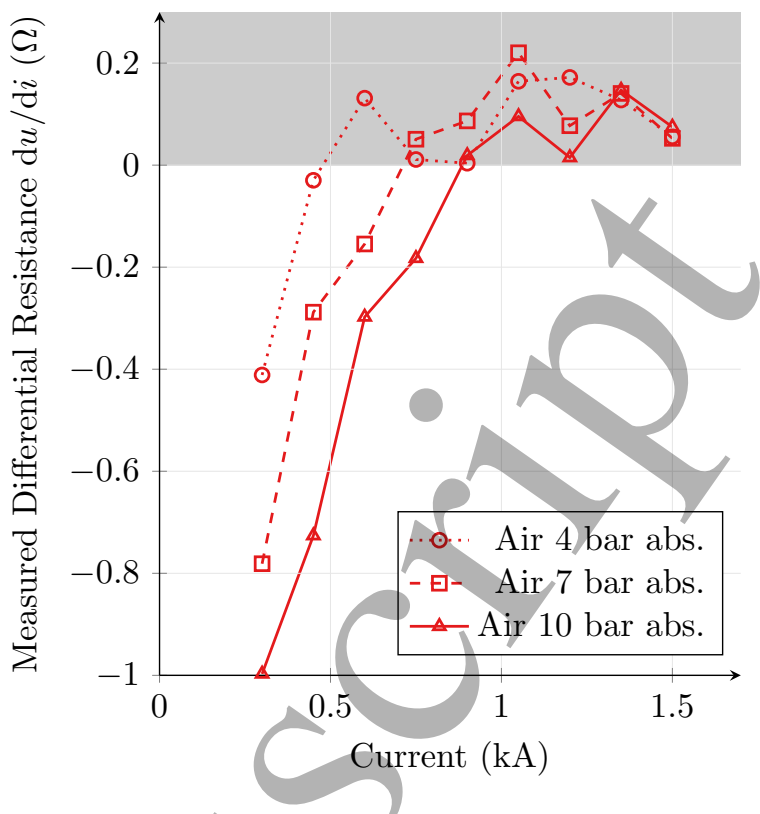

Figure 8: Experimentally obtained differential resistance $\mathrm{d} u / \mathrm{d} i$, for air at different pressures, as function of current. The unfavorable region where $\mathrm{d} u / \mathrm{d} i$ is positive is shaded in grey.

for passive oscillation switchgear. What matters for this DC switch topology is the absolute value of $\mathrm{d} u / \mathrm{d} i$ (more negative values are favourable), as well as the maximum current, where $\mathrm{d} u / \mathrm{d} i$ is still negative. Both improve with increasing pressure. Exact numbers of real devices are not known to the author, but parasitic resistance below $100 \mathrm{~m} \Omega$ seems realistic.

To interpret the experimental results the $1 \mathrm{D}$ model was used. The arc radius as function of position and pressure is shown in figure 4 . It shows a reasonable order of magnitude of a few $\mathrm{mm}$. Highspeed footage of the experiments is also available and shows comparable diameters of the light emitting zone. However, no quantitative information can be extracted from the videos without a more sophisticated optical setup. Currently, distortions by the lens effect of the PMMA nozzle and unclear definition of "arc boundary" introduce too many uncertain factors. With narrow bandpass filters it would be possible to determine radial temperature profiles like done by [17] for example. Similar measurements could be used as validation for the model. A simulated arc voltage can be obtained by integration the E-field (figure 6). Since the model breaks down in the downstream section, this was done only up to position $100 \mathrm{~mm}$, which is just upstream the throat and shown in figure 10b. These values are around $400 \mathrm{~V}$ to $600 \mathrm{~V}$, which agrees well with the experimental arc voltage up to the throat position as shown in [5], which were in the same range for the flat part of the $u(i)$ curve. 
From the simulation we learn that the arc radius scales with current squared, $r^{2} \propto i$, which is in agreement with Tuma and Lowke [6, 7]. In addition, we derive from our model that the arc radius cubed is inverse proportional to the pressure, i.e $r^{3} \propto 1 / p$, which is different from $[6,7]$, who found an even stronger dependence to the power of four. Lastly, the electric field scales with the square root of pressure, as can be seen in figure 6 and is again in agreement with Tuma and Lowke, as well as with the measurements.

As mentioned in section 2.2, the simulation results always show the arc voltage to be independent of current, as turbulent mixing is neglected. As soon as thermal conductance, including turbulence, has an effect on the central arc temperature, a negative differential arc resistance is expected [7]. Figure 9 shows the measured values of the differential resistance on the y-axis, plotted against the simulated arc radius for the respective current and pressure values on the $\mathrm{x}$-axis. It is clearly visible that there is a critical arc radius around $1.4 \mathrm{~mm}$, where $\mathrm{d} u / \mathrm{d} i$ goes to zero. This critical arc radius is independent of pressure and occurs at different current values for different pressures. In fact, for each pressure $p$ there is a current $i_{\text {crit }}$ which results in an arc radius of $r_{\text {crit }}$. These current values were found to scale with

$i_{\text {crit }} \propto p^{\frac{2}{3}}$,

as illustrated in figure 10c.

The existence of a critical radius can best be explained by the shear layer that separates the hot arc core from the cold gas flow around it. The author takes this value as an empirical measure for the thickness of the turbulent mixing layer. The model neglects this, but in reality this is definitively present. Lowke already describes this in [6], claiming that below $500 \mathrm{~A}$ their model did not give the same results as the experiments of Hermann [3]. For the nozzle shown in figure 1, the critical radius below which the arc core is getting too small to dominate the effects of the shear layer is $1.4 \mathrm{~mm}$. The nozzle profile with shorter converging section (red lines in figures) shows a smaller radius in the axial segment with highest acceleration (90 $\mathrm{mm}$ to $105 \mathrm{~mm}$ in figure 4), but slightly bigger values in the straight segments upstream. The voltage drop is highest in the segments with smallest radius. It might therefore be favourable to use a nozzle profile with a short converging section to get more negative $\mathrm{d} u / \mathrm{d} i$. Due to the resulting smaller arc radius, turbulence still influences the central arc temperature more, for otherwise equal conditions. This effect is illustrated in figure 10c. If the radius is evaluated not at $100 \mathrm{~mm}$ but at the position of its minimum (around $95 \mathrm{~mm}$ for the red lines shown in figure 4), the critical current is further increased. More detailed simulations and experiments are needed to

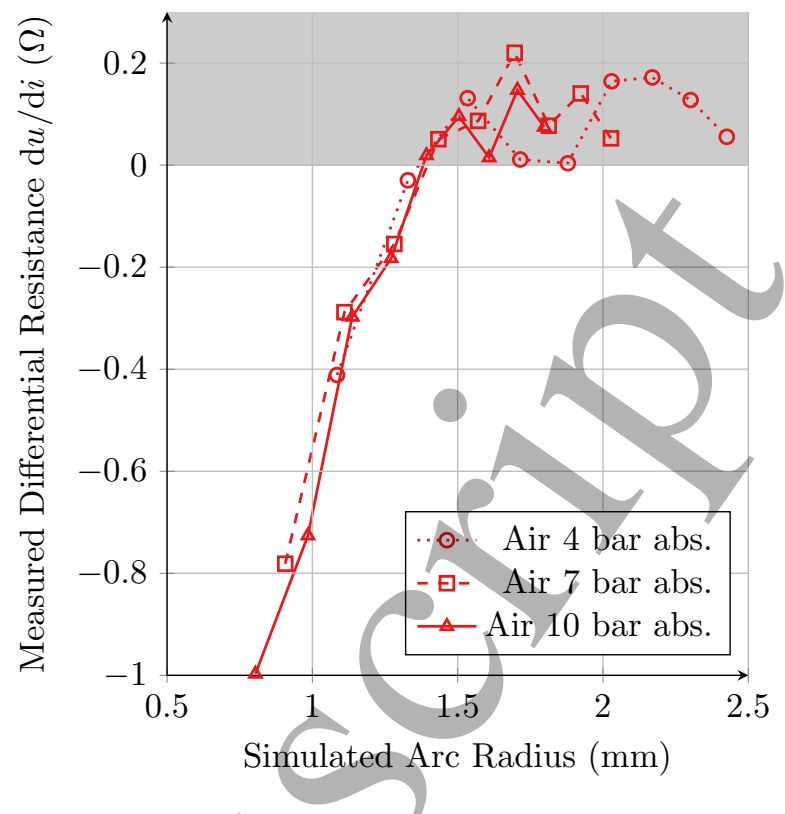

Figure 9: For each of the current and pressure combinations measured and plotted in Figure 8, the arc radius $5 \mathrm{~mm}$ upstream of the throat was calculated using the $1 \mathrm{D}$ model. Here, that simulated radius is used on the $\mathrm{x}$-axis, instead of the current as in figure 8 . The measured $\mathrm{d} u / \mathrm{d} i$ values for all pressures line up very well, with negative values corresponding to radii below $1.4 \mathrm{~mm}$, and unfavourable positive values above that critical radius.

quantitatively state the improvement due to the nozzle with shorter acceleration segment.

\section{Inlet conditions}

In section 4, turbulent mixing at arc boundary was assumed to be the major factor leading to negative $\mathrm{d} u / \mathrm{d} i$. This would mean the gas flow conditions play an important role. In an attempt to directly influence the turbulent shear layer (thickness), different pertubators were inserted in the cylindrical inlet section upstream of the nozzle.

\subsection{Experimental Results}

Figure 11 shows the resulting $u(i)$ curves. Almost all configurations show similar arc voltage. Only flow straightener number 1 showed slightly lower voltage, but with similar shape of the voltage curve.

\subsection{Discussion}

Although turbulent cooling of the plasma column is considered the main factor for a negative differential arc resistance, no change was observed due to the different inserts in the inlet segment. The inserts

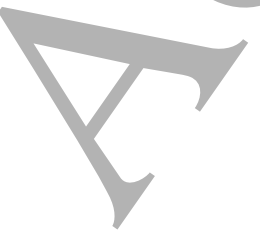


probably did not change the conditions at the shear layer between cold gas and hot plasma. The slightly lower voltage of fs1 most likely results from a pressure drop due to friction the long and narrow tubes.

\section{Blowing gas}

The experimental results in combination with the numerical model for air, as shown above, indicate that the ratio of arc radius and turbulent sheer layer determine at which current levels the differential arc resistance is negative. Thus, it is justified to assume that different blowing gases with different material parameters could also lead to different current levels at which the differential arc resistance changes from being negative to non-negative. This was analyzed experimentally, with three other gases. Nitrogen, carbon dioxide and helium where chosen as their main thermodynamic properties are quite different (see table 1 for values at standard conditions). As the setup is not sealed and the exhaust will be released to the environment, the use of other gases is restricted, particularly $\mathrm{SF}_{6}$ could not be used due to its global warming potential and toxic dissociation products.

\subsection{Experimental Results}

Figure 12 shows the influence of the blowing gas on the arc voltage. First, it can be noted that helium clearly gives the lowest arc voltage, and the least favourable $\mathrm{d} u / \mathrm{d} i$. For 7 bar, $\mathrm{d} u / \mathrm{d} i$ is positive starting from $500 \mathrm{~A}$, and for 10 bar it is zero form there on. Figure 13 shows the differential resistance $\mathrm{d} u / \mathrm{d} i$ for 10 bar as well.

\subsection{Discussion}

The results of the other gases are closer to each other, but differ still. Most surprising was the fact that nitrogen and air are so far apart. Depending on which exact value is still considered negative enough for the application, nitrogen might be the most favorable of the four gases, since its $\mathrm{d} u / \mathrm{d} i$ negative up to $1600 \mathrm{~A}$ (as can bee seen in figure 12), albeit the derivative is too close to zero to yield a reliable numeric result. $\mathrm{CO}_{2}$ is in between air and nitrogen, with lower overall voltage than $\mathrm{N}_{2}$, and $\mathrm{d} u / \mathrm{d} i$ negative up to $1000 \mathrm{~A}$, whereas air has positive $\mathrm{d} u / \mathrm{d} i$ starting at $800 \mathrm{~A}$.

\section{Conclusion}

The experimental results combined with the $1 \mathrm{D}$ model lead to several conclusions. First of all, ablation seems not to play a significant role for the given nozzle geometry and current values, at least not at a level where the $u(i)$ curve is dominated by the ablation process. Second, increasing the inlet pressure improves
Table 1: Properties of the different gases; air, carbon dioxide, helium, nitrogen [18]

\begin{tabular}{lcccc}
\hline Gas & $\begin{array}{c}\text { Speed of } \\
\text { Sound } \\
v_{\mathrm{s}}[\mathrm{m} / \mathrm{s}]\end{array}$ & $\begin{array}{c}\text { Density } \\
\rho\left[\mathrm{kg} / \mathrm{m}^{3}\right]\end{array}$ & $\begin{array}{c}\text { Thermal } \\
\text { Cond. } \\
k[\mathrm{~W} / \mathrm{m} \mathrm{K}]\end{array}$ & $\begin{array}{c}\text { Specific Heat } \\
{[\mathrm{kJ} / \mathrm{kgK}]}\end{array}$ \\
\hline $\mathrm{Air}$ & 343 & 1.21 & 0.0259 & 1.01 \\
$\mathrm{CO}_{2}$ & 267 & 1.84 & 0.0146 & 0.84 \\
$\mathrm{He}$ & 1008 & 0.17 & 0.142 & 5.19 \\
$\mathrm{~N}_{2}$ & 349 & 1.17 & 0.024 & 1.04 \\
\hline
\end{tabular}

the $u(i)$ curve for the application of passive oscillation switches, with a more than linear gain, see eq. (6). This is suspected to be due to the arc radius decrease, which was found to be proportional to $p^{1 / 3}$. The combination of experiments and model results indicate there seems to be a critical radius, above which $\mathrm{d} u / \mathrm{d} i$ is zero or positive, which is $1.4 \mathrm{~mm}$ in the given setup. Changing this critical radius by influencing the flow upstream of the arc did not prove successful.

Lastly, varying the blowing gas showed also quite some influence, on the general arc voltage, but also on the $\mathrm{d} u / \mathrm{d} i$, strengthening the hypothesis that mixing of cold and hot gas at the arc boundary and convective cooling of the arc core are the most likely reason for negative differential resistance.

\section{Outlook}

With further research effort it will certainly be possible to optimize gas blast circuit breakers for the application in passive oscillation DC switches. Specifically, on the experimental side, the arc radius and temperature are interesting variables that could be measured, for example with techniques shown by $[19,20]$. Increasing the pressure to higher values is also interesting, in order to see if there is a limit at which further increasing does not yield a better $u(i)$ behavior. Going to higher pressures was not possible with the FPDCS, mainly because of voltage and energy constraints.

The same goes for higher current. Testing up to at least $5 \mathrm{kA}$ to $8 \mathrm{kA}$ would be required to cover realistic load- or over-current situations. Both should be possible after an upgrade of the FPDCS, which is currently being developed at ETH. With a more powerful source, it might also be possible to test performance with an LC-circuit attached, which would give an insight into how negative the differential resistance really needs to be, and how fast the oscillating current is amplified by a real arc. Especially the influence of fluctuations around the median arc voltage are of interest there.

Regarding the modelling, the current implementation tends to get unstable in the converging segments 
above 20 bar, and as described above, the diverging part did not yield useable results. Both issues could be addressed by adapting the boundary conditions and adding a constraint enforcing the second law of thermodynamics, as well as by improving the minimization algorithm to avoid getting stuck in local minima. Others are also developing more sophisticated models that include radiation transport and fluid dynamics more accurately, like for example [21, 22] or more recently [23]. Using these models probably gives a much more accurate picture of the arc, and might prove (or disprove) the hypothesis of the critical radius.

\section{Acknowledgment}

The authors would like to thank ABB Switzerland for financial support. This research is part of the activities of the Swiss Centre for Competence in Energy Research on the Future Swiss Electrical Infrastructure (SCCERFURIES).

\section{References}

[1] Franck C M, Smeets R and members A3/B434 2017 Cigre Technical Brochure 683 - Technical Requirements and Specifications of State-of-the-Art HVDC Switching Equipment Tech. Rep. April JWG A3/B4,34

[2] Lee A W R C, Slade P G W R C, Yoon K H W P, Porter J E S D, EPRI) and Vithayathil J B P A 1985 IEEE Transactions on Power Apparatus and Systems PAS104 2721-2729 ISSN 0018-9510 URL https://doi.org/ 10.1109/TPAS.1985.319113

[3] Hermann W, Kogelschatz U, Niemeyer L, Ragaller K and Schade E 1974 Journal of Physics D: Applied Physics 7 1703-1722 ISSN 00223727 URL https://doi.org/ 10.1088/0022-3727/7/12/317

[4] Bort L S J, Freiermuth V and Franck C M 2017 Plasma Physics and Technology 4 1-4 URL https://doi.org/ 10.14311/ppt.2017.2.145

[5] Bort L S J and Franck C M 2019 IEEE Transactions on Plasma Science 47 714-720 ISSN 0093-3813 URL https://doi.org/10.1109/TPS.2018.2880950

[6] Tuma D T and Lowke J J 1975 Journal of Applied Physics 46 3361-3367 ISSN 00218979 URL https://doi.org/ 10.1063/1.322240

[7] Lowke J J and Ludwig H C 1975 Journal of Applied Physics 46 3352-3360 ISSN 0021-8979 URL https://doi.org/ 10.1063/1.322239

[8] Bort L S J and Franck C M 2016 Effects of nozzle and contact geometry on arc voltage in gas circuitbreakers 2016 IEEE International Conference on High Voltage Engineering and Application (ICHVE) (IEEE) pp 1-4 ISBN 978-1-5090-0496-6 URL https://doi.org/ 10.1109/ICHVE. 2016.7800700

[9] Ritter A, Bort L and Franck C 2016 Five years of pulsed current testing for HVDC switchgear 2016 IEEE International Conference on High Voltage Engineering and Application (ICHVE) (IEEE) pp 1-4 ISBN 978-1-5090-0496-6 URL https : // doi.org/10.1109/ ICHVE.2016.7800658

[10] D'Angola A, Colonna G, Gorse C and Capitelli M 2007 The European Physical Journal D 46 129-150 ISSN 1434-6060 URL https ://doi.org/10.1140/epjd/e200700305-4
[11] D'Angola a, Colonna G, Gorse C and Capitelli M 2011 The European Physical Journal D 65 453-457 ISSN 1434-6060 URL https://doi.org/10.1140/epjd/e201120424-5

[12] Aubrecht V and Bartlova M 2009 Plasma Chemistry and Plasma Processing 29 131-147 ISSN 0272-4324 URL https://doi.org/10.1007/s11090-008-9163-x

[13] Christen T 2006 Journal of Physics D: Applied Physics 39 4497-4503 ISSN 0022-3727 URL https://doi.org/ 10.1088/0022-3727/39/20/03

[14] Panousis E, Bujotzek M and Christen T 2014 IEEE Transactions on Power Delivery 29 1806-1813 ISSN 0885-8977 URL https:// doi.org/10.1109/ TPWRD. 2014.2312134

[15] Hermann W, Kogelschatz U, Ragaller K and Schade E 1974 Journal of Physics D: Applied Physics 7 607-619 ISSN 00223727 URL https://doi.org/10.1088/0022-3727/7/ $4 / 315$

[16] Walter M M 2013 Switching Arcs in Passive Resonance HVDC Circuit Breakers Ph.D. thesis ETH Zürich URL https://doi.org/10.3929/ethz-a-010112102

[17] Panousis E, Stoller P C, Carstensen J, Teppati V, Methling R, Franke S and Gorchakov S 2017 Active and passive optical diagnostics in a model HV circuit breaker XXXIII ICPIG vol 33 p 1

[18] Engineering ToolBox https : / / www.engineeringtoolbox.com [Online; accessed March $16,2018]$

[19] Franke S, Methling R, Uhrlandt D, Bianchetti R, Gati R and Schwinne M 2014 Journal of Physics D: Applied Physics 47 015202 ISSN 0022-3727 URL https:// doi.org/10.1088/0022-3727/47/1/015202

[20] Stoller P C, Panousis E, Carstensen J, Doiron C B and Färber R 2015 Journal of Physics D: Applied Physics 48 015501 ISSN 0022-3727 URL https://doi.org/10.1088/ 0022-3727/48/1/015501

[21] Nordborg H and Iordanidis A A 2008 Journal of Physics D: Applied Physics 41135205 ISSN 0022-3727 URL https://doi.org/10.1088/0022-3727/41/13/135205

[22] Iordanidis A A and Franck C M 2008 Journal of Physics D: Applied Physics 41 135206 ISSN 0022-3727 URL https://doi.org/10.1088/0022-3727/41/13/135206

[23] Fuchs R, Murmann M and Nordborg H 2017 Plasma Physics and Technology Journal 4 79-82 ISSN 23362634 URL https://doi.org/10.14311/ppt.2017.1.79 


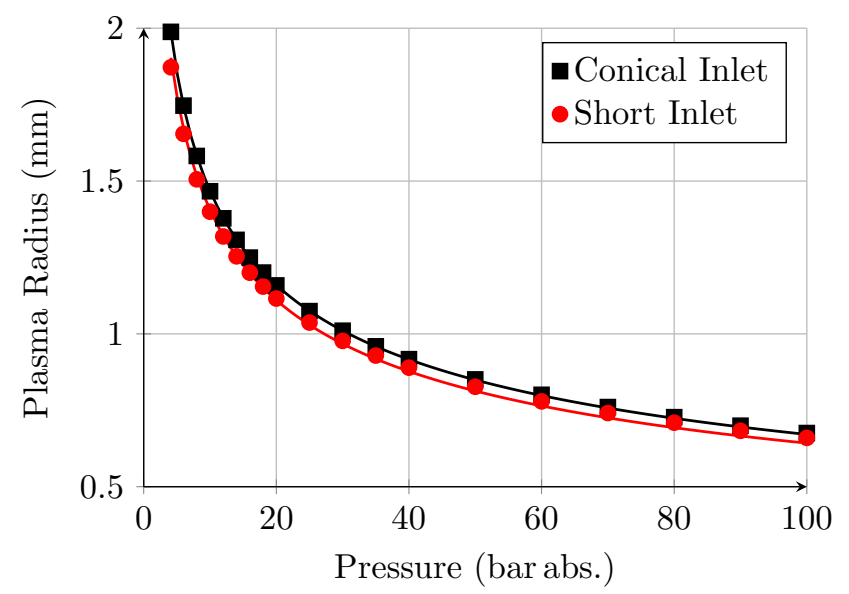

(a) Arc radius at $100 \mathrm{~mm}$ as function of pressure. Marks are simulation results, the solid lines show $r \propto 1 / p^{-3}$ fits.

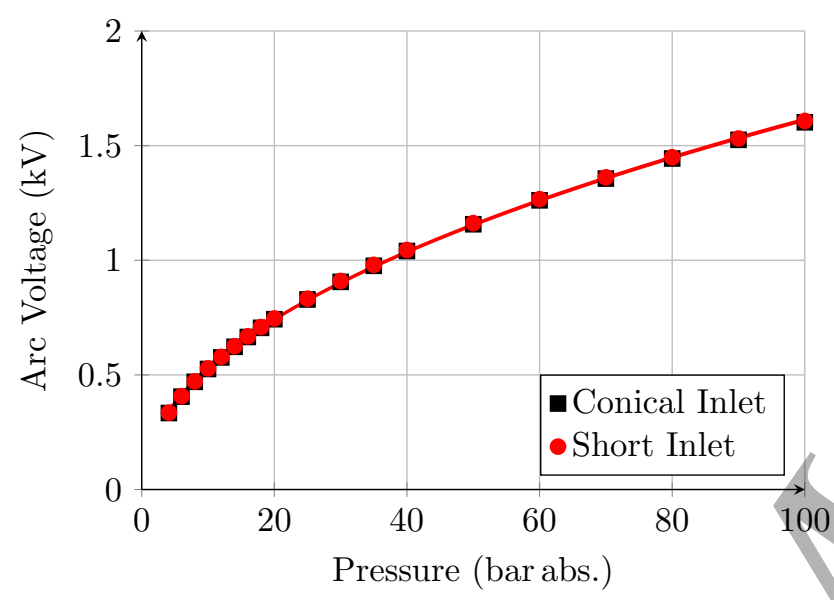

(b) Arc voltage as function of pressure, with the current fixed at $1000 \mathrm{~A}$. Obtained by integrating the E-field from $35 \mathrm{~mm}$ to $100 \mathrm{~mm}$. Marks show simulation results, solid lines $u \propto p^{0.5}$ fits of these results.

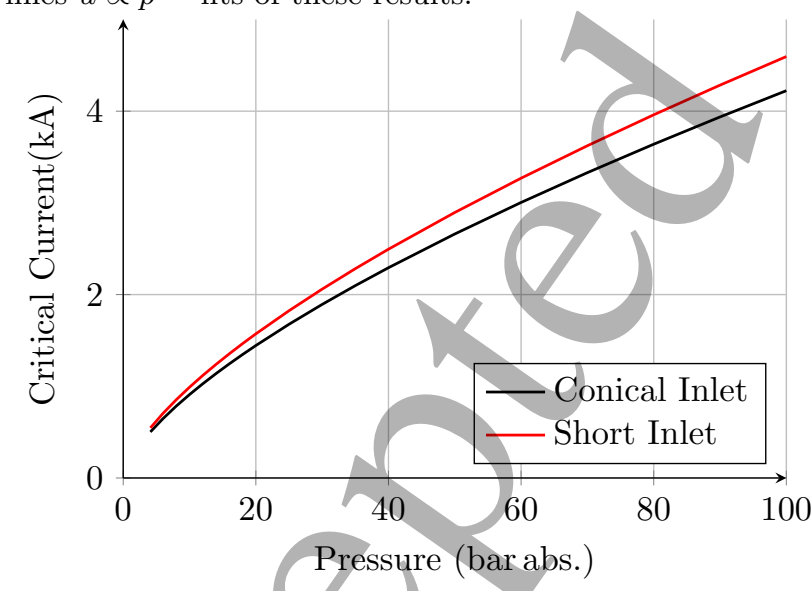

(c) Critical Current, i.e. the current leading to an arc radius of $1.4 \mathrm{~mm}$, as function of pressure. Lines are obtained from $i_{\text {crit }} \propto p^{2 / 3}$.

Figure 10: 1D-model simulation results, for varying pressure and the two nozzles shown in figure 1.

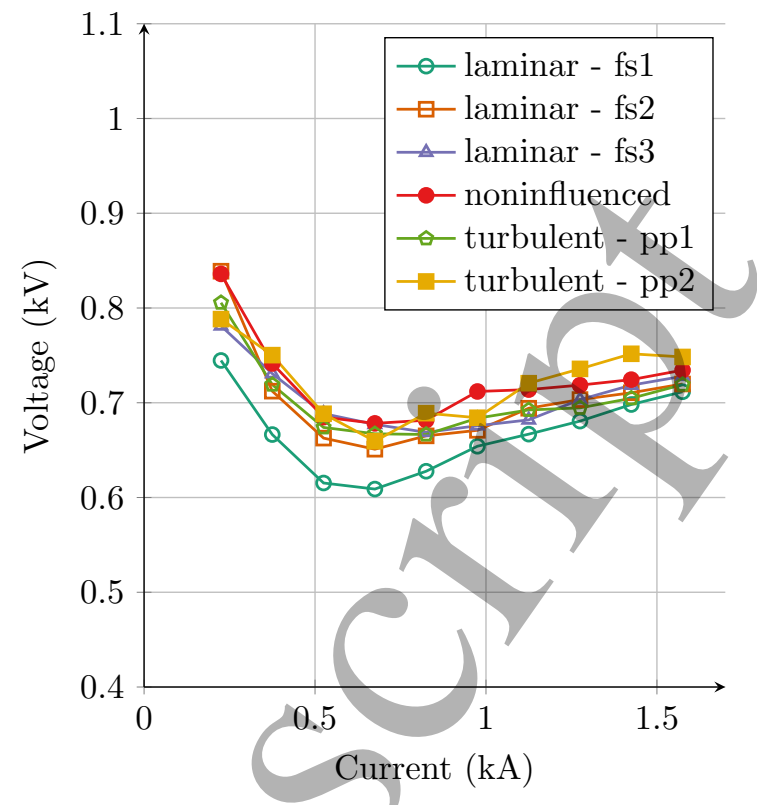

Figure 11: Measured arc voltage as function of current for experiments with different inserts in the gas flow. Inlet pressure is 8 bar upstream the nozzle.

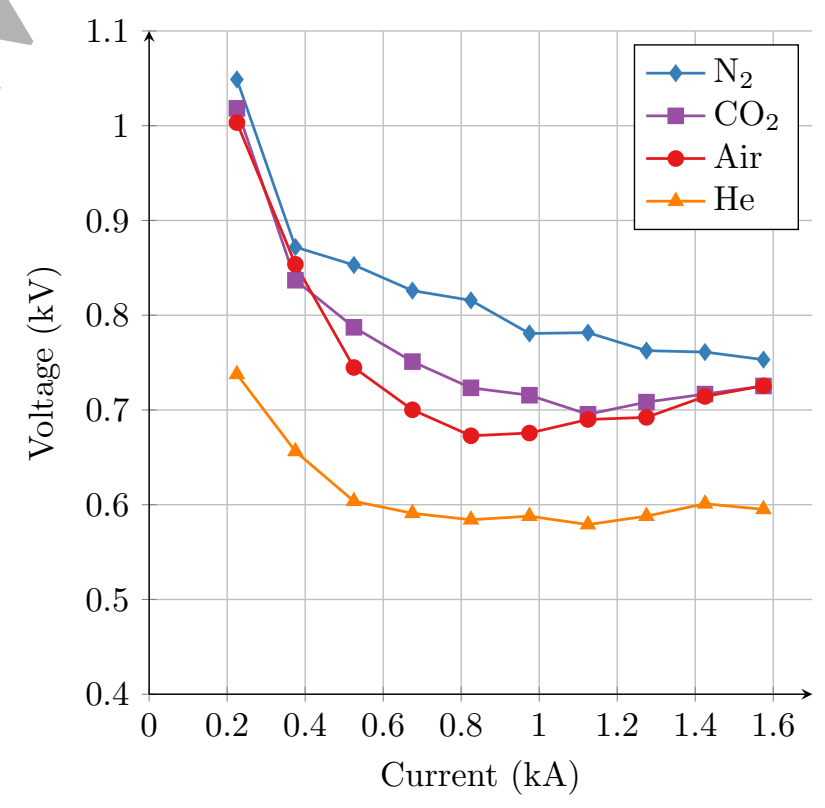

Figure 12: Measured arc voltage as function of current for experiments with different gases at inlet pressure of 10 bar 


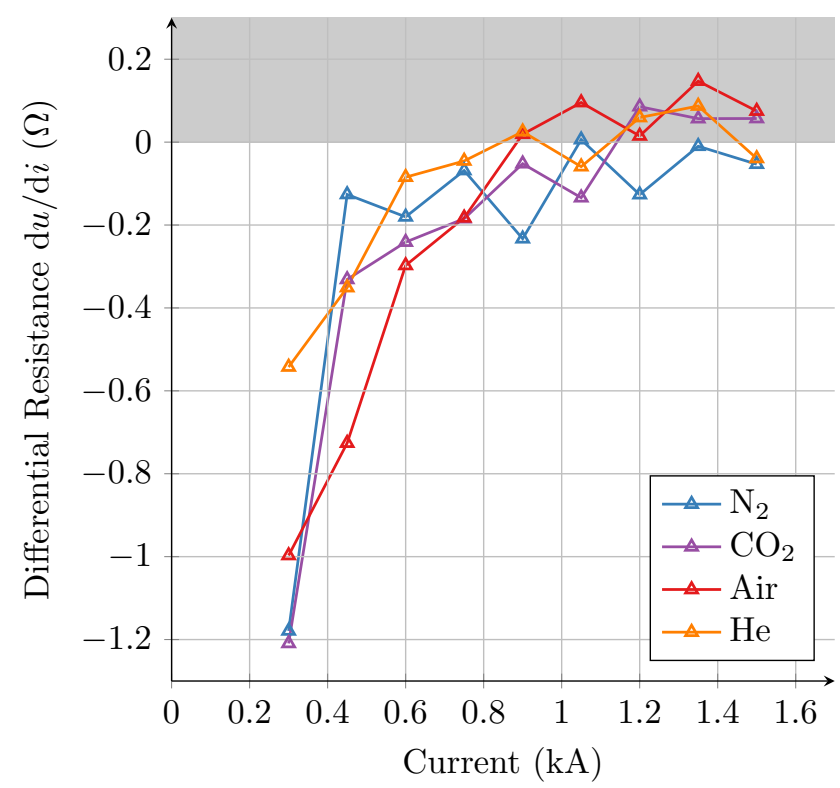

Figure 13: Experimentally obtained differential resistance $\mathrm{d} u / \mathrm{d} i$ as function of current for the different gases. Inlet pressure is $10 \mathrm{bar}$ in all cases. As in figures 8 and 9 , the unfavorable area of positive $\mathrm{d} u / \mathrm{d} i$ is shaded grey. 